\title{
Cardiac rehabilitation in patients with heart failure and diabetes mellitus
}

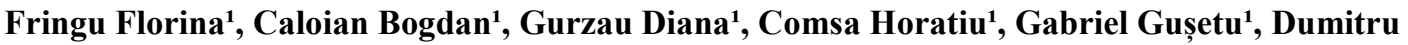 \\ Zdrenghea $^{1}$, Dana Pop ${ }^{1}$
}

1.Cardiology - Rehabilitation Department, "Iuliu Hatieganu"University of Medicine and Pharmacy ”, Cluj-Napoca

\begin{abstract}
Over the past decades, cardiac rehabilitation has had a spectacular evolution, from the mere monitoring of the patients' return to physical activity to a multidisciplinary approach focused on patient education, individualized physical exercise, changes in cardiovascular risk factors and, not least, an improvement in patient quality of life. Heart failure represents an important public health problem, and the association of this disease with diabetes mellitus significantly reduces prognosis in these patients, the two disorders potentiating each other. Recent data demonstrate a significant benefit of cardiac rehabilitation in patients with diabetes mellitus and heart failure, with important effects in reducing mortality, increasing exercise capacity and improving symptoms. Unfortunately, cardiac rehabilitation is generally underused, most probably due to the lack of awareness and low adherence of patients, as well as due to insufficiently developed programs at national level.
\end{abstract}

Key words: cardiovascular rehabilitation, diabetes mellitus, heart failure

Heart failure is an important public health problem both at national and international level, affecting 1$2 \%$ of the adult population worldwide, with a greater predisposition of elderly subjects because of the multiple associated comorbidities [1].

This represents the possible final evolution of any cardiac disease which, despite complex treatments, frequently affects patient quality of life in a significant manner and results in very high mortality rates. The majority of patients with heart failure have multiple cardiovascular risk factors. A special category of patients is represented by those with heart failure and diabetes mellitus [2,3].

Type 2 diabetes mellitus most often coexists with other risk factors such as hypertension, dyslipidemia, smoking, obesity, which are determinant factors of atherosclerosis, ischemic heart disease and left ventricular dysfunction. At the same time, it is known that diabetes may also cause heart failure independently of coronary disease, through diabetic cardiomyopathy [4]. Epidemiological studies show that the incidence of heart failure is 2.5 times higher in the diabetic population compared to non-diabetic subjects, and approximately $40 \%$ of heart failure patients with low ejection fraction are diabetic [5]. Thus, diabetes mellitus is one of the most frequent comorbidities found in patients with heart failure, and these figures continuously increase with the aging of the population [3].

The evolution and prognosis of diabetic patients with heart failure are more unfavorable than in those without this comorbidity. Also, mortality in these patients remains high even if new therapies have improved this percentage over the past years [5]. Thus, it was demonstrated that 5-year survival is 50\% and 10 -year survival is $10 \%$ after the diagnosis of heart failure [7]. This is why, in addition to drug therapy indicated by guidelines, an essential role in their long-term approach is played by cardiovascular rehabilitation programs.

The aim of this article was to perform an analysis of the literature data assessing cardiac rehabilitation in diabetic patients with heart failure, and to highlight indications, benefits and the most common problems encountered in this process.

In general, cardiac rehabilitation is divided into three phases, all being aimed at an optimal recovery of the patient and at the prevention of subsequent cardiovascular events. Phase 1 (inpatient phase) is initiated during hospitalization, and consists of the patient's progressive mobilization for the performance of usual activities. This phase also involves counseling on the etiology, treatment, risk factors of the disease, as well as the subsequent follow-up plan.

Phase 2 involves an outpatient supervised rehabilitation program for a period of 3-6 months, consisting of physical exercise, further approach of risk factors, and pharmacological and nonpharmacological secondary prevention measures.

Phase 3 of rehabilitation is represented by physical exercise performed at home, monitoring of risk factors, and application of lifestyle changing measures. It is indicated throughout life $[8,9]$. 
Cardiac rehabilitation in patients with heart failure has certain particularities. This category of patients have a significantly reduced exercise capacity, which may result in a decrease of daily activities and consequently, an impairment of quality of life.

Cardiac rehabilitation seems to be a good solution to improve quality of life and ameliorate the prognosis of patients suffering from heart failure [10].

Until the past few years, physical restrictions were imposed in the case of heart failure patients, the indications for cardiac rehabilitation in this category of patients being quite recent and limited to patients with stable compensated heart failure, NYHA II and III, without significant cardiac arrhythmias [11].

The trial Heart Failure-A Controlled Trial Investigating Outcomes of Exercise Training (HFACTION), published in 2015, aimed to evaluate the safety and efficacy of physical training in patients with NYHA II-IV heart failure, under optimal treatment. It was demonstrated that exercise training is safe in this category of patients and leads to a moderate reduction of cardiovascular events [12].

A Cochrane analysis of 33 trials including patients with NYHA II and III heart failure showed an improvement of the hospitalization rate and exercisebased cardiac rehabilitation independently of the type of rehabilitation program, as well as a reduction of the mortality rate in these patients at a follow-up of $>1$ year [13].

Another study that analyzed the prognosis of heart failure patients included in cardiac rehabilitation programs demonstrated that a $5 \%$ improvement of predicted fitness led to a $10 \%$ decrease in the risk of hospitalization and all-cause mortality [14].

Cardiac rehabilitation in diabetic patients has certain particularities. Diabetic patients present, in addition to cardiac impairment, peripheral and autonomic dysfunction, which contributes to the diminution of exercise capacity and tolerance. These problems seem to be more significant in insulin-dependent patients. Furthermore, it was demonstrated that diabetic patients with heart failure are less compliant with the rehabilitation program and have a higher risk of hospitalization [15].

It was also demonstrated that this category of patients have a more limited response to physical exercise compared to non-diabetic patients, independently of the adherence to the rehabilitation program. This is why a complex approach, focused on a strict diet, compliance with the rehabilitation program and adherence to treatment, is extremely important in this category of patients $[16,17]$.

The contraindications of cardiac rehabilitation are limited to the physical exercise part of the program, all the other measures being indicated for all patients, without restriction. These include patients with unstable angina, decompensated heart failure, ventricular arrhythmias, pulmonary hypertension, with values higher than $60 \mathrm{mmHg}$, intracardiac thrombi, recent thrombophlebitis with or without thromboembolism, severe obstructive cardiomyopathy, tight aortic stenosis, inflammatory or infectious diseases, and musculoskeletal diseases which prevent performing physical exercise [18].

The benefits of cardiac rehabilitation are the result of all the program components. Thus, about half of the benefit on mortality is due to the reduction of risk factors, of which the most important is smoking.

Another important benefit is the improvement of exercise tolerance by a $33 \%$ increase of tolerated metabolic equivalents and a $16 \%$ increase of maximum oxygen consumption. The quality of life of these patients is also improved by a reduction of symptoms, a diminution of stress and depression $[19,20]$.

A meta-analysis including 9 randomized trials confirmed a $35 \%$ reduction of mortality in this category of patients [13].

A large randomized trial, ACTION HF, which included 2331 patients, demonstrated a 15\% decrease of all-cause mortality, cardiovascular mortality and hospitalization for heart failure after a rehabilitation program followed up over 2.5 years. It should be mentioned that positive results were not obtained on initial analysis, but only after adjustment for prognostic criteria [12].

In diabetic patients, studies demonstrate benefits through a delay in cardiovascular manifestations, an increase of exercise capacity and a reduction in the number of hospitalizations. Also, in patients at high risk for the development of diabetes, physical exercise reduces this risk by $25-50 \%$ [17]. The most important mechanisms involved are an increase of insulin sensitivity, a decrease of blood pressure, a reduction of visceral adiposity, an improvement of endothelial function, a diminution of vascular stiffness and systemic inflammation [17].

In conclusion, the cardiac rehabilitation of diabetic patients with heart failure is a real challenge for both doctors and patients, provides significant benefits 
demonstrated by recent studies, and should be implemented as often as possible.

References

1. Ausili D, Rebora P, Mauro SD, et al. Clinical and socio-demographic determinants of self-care behaviours in patients with heart failure and diabetes mellitus: A multicentre cross-sectional study. International Journal of Nursing Studies. 2006; 63, 18-27.

2. Howlett JG, Macfadyen JC. Treatment of Diabetes in People with Heart Failure. Canadian Journal of Diabetes. 2013; Suppl 1:S126-8

3. Cas AD, Fonarow GC, Gheorghiade $\mathrm{M}$. Concomitant Diabetes Mellitus and Heart Failure. Current Problems in Cardiology. 2015; 40(1), 7-43.

4. Sitar -Tăut AV, Pop D, Zdrenghea DT. NTproBNP values in elderly heart failure patients with atrial fibrillation and diabetes. J Diabetes Complications. 2015;29(8):1119-23.

5. Nichols GA, Hillier TA, Erbey JR, et al. Congestive heart failure in type 2 diabetes: prevalence, incidence, and risk factors. Diabetes Care. 2001;24:1614-1619.

6. Roger V. Epidemiology of Heart Failure. Circulation Research. 2013; 113(6):646-659.

7. Mosterd A, Cost B, Hoes AW, et al. The prognosis of heart failure in the general population: The Rotterdam Study. Eur Heart J. 2001;22:1318-1327.

8. Squires RW, Gau GT, Miller TD, et al. Cardiovascular rehabilitation: status, 1990. Mayo Clin Proc. 1990;65:731-55.

9. Balady GJ, Williams MA, Ades PA, et al. Core Components of Cardiac Rehabilitation/Secondary Prevention Programs: 2007 Update: A Scientific Statement From the American Heart Association Exercise, Cardiac Rehabilitation, and Prevention Committee, the Council on Clinical Cardiology; the Councils on Cardiovascular Nursing, Epidemiology and Prevention, and Nutrition, Physical Activity, and Metabolism; and the American Association of Cardiovascular and Pulmonary Rehabilitation. Circulation. 2007;115(20):2675-2682.

10. Pop D, Caloian B, Cismaru G, et al. Secondary prevention- an essential component of the comprehensive rehabilitation of patients with heart failure. Balneo Research Journal. 2017;8(4):217-219
11. Sullivan MJ, Higginbotham MB, Cobb FR. Exercise training in patients with severe left ventricular dysfunction. Hemodynamic and metabolic effects. Circulation. 1988;78:506-15.

12. O'Connor CM, Whellan DJ, Lee KL, et al. Efficacy and safety of exercise training in patients with chronic heart failure: HF-ACTION randomized controlled trial. JAMA 2009;301:1439-50.

13. Piepoli MF, Davos C, Francis DP, et al. Exercise training meta-analysis of trials in patients with chronic heart failure (ExTraMATCH). BMJ 2004; 328(7433):189.

14. Sabbag A, Mazin I, Rott D, et al. The prognostic significance of improvement in exercise capacity in heart failure patients who participate in cardiac rehabilitation programme. European Journal of Preventive Cardiology. 2018; 25(4):354-361.

15. Greenberg BH, Abraham WT, Albert NM, et al. Influence of diabetes on characteristics and outcomes in patients hospitalized with heart failure: a report from the Organized Program to Initiate Lifesaving Treatment in Hospitalized Patients with Heart Failure (OPTIMIZE-HF). American heart journal. 2007; 154:277.e1277.e8.

16. Keteyian SJ, Leifer ES, Houston-Miller N, et al. Relation between volume of exercise and clinical outcomes in patients with heart failure. Journal of the American College of Cardiology. 2012; 60:1899-1905.

17. Zdrenghea D, Pop D, Penciu O, et al. Rehabilitation in diabetic patients. Rom J Intern Med. 2009; 47(4):309-17.

18. Naughton J. Exercise training for patients with coronary artery disease. Cardiac rehabilitation revisited. Sports Med. 1992;14:304-19

19. Taylor RS, Unal B, Critchley JA, et al. Mortality reductions in patients receiving exercise-based cardiac rehabilitation: how much can be attributed to cardiovascular risk factor improvements? Eur J Cardiovasc Prev Rehabil. 2006;13:369-74

20. Maines TY, Lavie CJ, Milani RV, et al. Effects of cardiac rehabilitation and exercise programs on exercise capacity, coronary risk factors, behavior, and quality of life in patients with coronary artery disease. South Med J. 1997;90:43-9. 